\title{
Д.И. Небольсин
}

\section{ДИСЦИПЛИНАРНЫЕ И ТЕОРЕТИЧЕСКИЕ ОСОБЕННОСТИ АНАЛИТИЧЕСКОЙ ФИЛОСОФИИ ИЗОБРАЖЕНИЙ}

\begin{abstract}
Рассматриваются основные признаки дисциплинарной и методологической идентичности аналитической философии изображений с иелью определить ее отличия от других школ изучения визуального. В качестве наиболее значимых отличий выявляются следующие: ограничение исследовательской проблематики областью изобразительной репрезентации, отмежевание от нормативной эстетики, методологическое разнообразие и работа с феноменологическими и онтологическими вопросами посредством уточнения понятийного аппарата.

Ключевые слова: репрезентация, изображения, аналитическая философия, теория образа.
\end{abstract}

Искусствоведение и история искусства, иконология, визуальные исследования, семиотика, теория образа, арт-критика, визуальная социология таков неполный список школ, дисциплин и методологий, затрагивающих проблематику визуального и образного. Эта обширная область не поддается беглому картографированию, и связанные с ней дискуссии порой приводят к формированию достаточно необычных исследовательских программ, таких как аналитическая философия изображений. Поскольку на сегодня она практически не представлена в отечественной научной литературе, цель данной статьи - выделить ключевые вопросы, понятия и установки, общие для этого неоднородного направления исследований, в котором практически нет наглядных самоописаний.

Само обозначение «аналитическая философия изображений» (далее АФИ) не очень распространено ${ }^{1}$ и используется нами по аналогии с другими тематическими ответвлениями аналитической философии, такими, например, как аналитическая философия языка или аналитическая философия истории. Представители АФИ придерживаются характерного стиля письма, сочетающего строгость и ясность аргументации с полемической направленностью, но сравнительно редко обращаются к классикам вроде Бертрана Рассела или Уилфрида Селларса. При этом в АФИ не практикуется историческая реконструкция дискуссий в ее рамках, а функцию специализированных учебников или ридеров выполняют либо сборники статей $[1,2]$, либо монографии, которые можно назвать обзорными лишь условно, поскольку их авторы сопровождают изложение имеющихся концепций их развернутой критикой и экспликацией собственных подходов. Примерами книг такого рода могут служить «Понимание изображений» Доминика М. Лопеса [3] и «Образы» Джона Кульвицки [4]. Вместе с тем почти каждая серьезная (т.е. содержащая

\footnotetext{
${ }^{1}$ Представители АФИ редко оговаривают свою принадлежность к аналитической философии, предпочитая обозначения вроде «философия изображений (philosophy of depiction)», «теория репрезентации» и проч.
} 
оригинальную и аргументированную теорию) монография в этой области не обходится без полемического обзора альтернативных концепций.

По меньшей мере один из центральных для АФИ вопросов нетрудно предугадать: «что такое изображение?» Во избежание разногласий на уровне центральных понятий этот термин трактуется предельно просто: им обозначается любой объект, подпадающий под обыденное понимание слова «изображение». Очевидные примеры - рисунки или фотографии, т.е. все, что обладает некоторым материальным носителем (будь то холст, бумага или стена) и некоторым фигуративным или нефигуративным ${ }^{1}$ визуальным содержанием. Проще говоря, изображение - это картинка, а не образ, поскольку образ может не иметь материального носителя и быть ментальным, оптическим, тактильным или, например, звуковым [5. Р. 9-14]. Разумеется, картинки тоже неоднородны - к ним относятся не только фотографии в семейных альбомах или полотна в музеях, но и инженерные чертежи, фотороботы, портреты звезд на футболках или рисунки на коробках конфет. Разнообразие изображений (pictorial diversity) подталкивает к постановке вопроса о том, что общего есть у этих артефактов помимо их визуальной специфики. В АФИ на него отвечают примерно так: все они репрезентируют что-то, однако особым способом - изображения невозможны без депикции (depiction) или изобразительной репрезентации (pictorial representation). Этот тезис с неизбежностью провоцирует дальнейшие вопросы, а также заставляет остановиться на одном существенном терминологическом затруднении.

Оно связано с тем, что pictorial representation и depiction настолько тесно переплетенные понятия, что в первом приближении их можно посчитать синонимичными. Но в АФИ они, как правило, не признаются таковыми: «картина может изображать (to depict) агнца, и агнец может репрезентировать (to represent) Христа; но картина не изображает Христа» [6. Р. 383]. Этот пример, предложенный Кристофером Пикоком, недостаточно точен (в нем не говорится именно о пикториальной репрезентации, которая в данном случае выполняла бы те же функции, что и депикция), но он демонстрирует, что репрезентация - это исключительно широкое, расхожее и неопределенное понятие, использование которого при объяснении изображений может привести к возникновению целого ряда трудностей. Депикция - термин с более узким значением, но в нем имплицирован фигуративный характер изображений, в то время как далеко не все из них фигуративны. Оба варианта обозначения проблематичны: они легко могут привести к объяснению изображений по модели реалистической живописи или фотографии либо же к ограничению этого объяснения спецификой отношений изображений с их референтами. Но в АФИ подобным стратегиям противостоят альтернативные - к примеру, трактующие изобразительную репрезентацию не как копирование воспроизводства, а как интерпретацию видимого мира [7. Р. 31-33]. В этом случае подчеркивается, что изображения асимметричны как предмету, так и обыденному перцептивному опыту. То есть базовое теоретическое допущение АФИ о репрезентативном характере изображений не только ограничивает

\footnotetext{
${ }^{1}$ Вопрос о том, являются ли абстракции предметом интереса АФИ, остается дискуссионным: если одни авторы уделяют им специальное внимание либо включают их в область охвата своих теорий, то другие отрицают их принадлежность к классу изображений. Первая позиция встречается чаще, но и её сторонники склонны уделять больше внимания фигуративным изображениям.
} 
затрагиваемую ею проблематику, но и допускает множественность вариантов работы с этим допущением.

Приведем список вопросов, которые могут прояснить специфику и неоднородность АФИ (далеко не каждая из связанных с ней теорий затрагивает все эти вопросы или ограничивается ими $)^{1}$ :

(1) Что такое изображение? Приведенная выше отсылка к повседневному использованию этого термина не дает ответа: как правило, она служит отправной точкой для дальнейшей работы.

(2) Что такое изобразительная репрезентаџия/депикиия? Этот вопрос можно уточнить следующим образом. Допустим, перед нами иллюстрация из учебника по анатомии. Почему мы видим в ней именно схему поперечного разреза головного мозга, а не монохромную абстракцию или фотографию кота?

(3) Чем изображения отличаются от других репрезентаций? Хотя аналитическая традиция плотно ассоциируется с лингвоцентризмом, в АФИ принято избегать редукционистских объяснений.

(4) Как объяснить онтологическую двойственность изображений? В любом изображении можно выделить: (а) материальную плоскость с пятнами, линиями, точками, мазками и прочими метками (marks) и (б) нематериальный объект изображения (репрезентируемые вещи, события, состояния и т.д.), который чаще всего и привлекает наше внимание. Объяснение соотношений этих элементов представляется одной из наиболее сложных задач, которые ставят перед собой теоретики АФИ.

(5) Каковы необходимые и достаточные условия базового понимания изображений? Термин «понимание» в этом случае трактуется в минималистическом ключе: если мы видим кота в фотографии кота, то фотография нами понята. Но как раскрыть механику этого понимания, не прибегая к семиотическому редукционизму или предположению, что изображения объективно похожи на свои объекты?

(6) Что представляет собой опыт восприятия изображений (pictorial experience)? Как правило, восприятие изображений коренным образом отличается от восприятия предметов лицом к лицу. При этом важно, что прояснение разницы между ними по возможности не должно приводить к перцептуализму, т.е. редукции теории изображений к теории их восприятия.

Многие из этих вопросов (в первую очередь те, что затрагивают онтологическую двойственность и восприятие изображений) были впервые поставлены философами искусства в 60-70-е г. XX в. [7; 11]. Дальнейшие обсуждения этих проблем привели к формированию АФИ как достаточно автономной в теоретическом отношении школы, обладающей, впрочем, неустойчивым дисциплинарным статусом: до сих пор зачастую ее считают подразделом эстетики. Это обусловлено не только ее генезисом: работы по философии изображений регулярно публикуются в British Journal of Aesthetics и Journal of Aesthetics and Art Criticism, а краткие обзоры теорий изобразительной репрезентации встречаются во многих заметных антологиях и учебных пособиях по философии искусства [11-15]. Профессиональные траектории представи-

\footnotetext{
${ }^{1}$ Вопросы 1, 2, 5 и 6 даны в формулировках, близких к предложенным Робертом Хопкинсом [8]. Вопрос 3 затрагивается главным образом структурными теоретиками (см. ниже). Вопрос 4 восходит к работам Майкла Поланьи [9] и Майкла Подро [10].
} 
телей АФИ также во многих случаях связаны с эстетикой - в качестве примера можно привести отрывок из интервью с Домиником М. Лопесом: «...моя учеба в докторантуре была почти полностью ограничена философией языка и философией сознания; я толком не представлял, что такое эстетика... Потом я написал эту книгу - «Понимание изображений» - и переехал в Штаты, где все сказали: «Да ты занимаешься эстетикой», на что я ответил: «Да? Ну ладно!» и стал посещать собрания Американского общества по эстетике. Но было бы несколько необоснованно утверждать, что каждый, кто занимается изображениями, занимается эстетикой» [16. Р. 1-2].

С последним замечанием нельзя не согласиться: если некоторых представителей АФИ (К. Уолтон, Р. Уоллхейм и др., включая позднего Лопеса) можно без особых сомнений причислить и к философам искусства, то к другим (Дж. Кульвицки, Ф. Шиер и т.д.) эта характеристика неприменима, поскольку эти авторы либо не затрагивают проблематику искусства, либо делают это исключительно редко. Но важнее то, что убедительная теория изобразительной репрезентации должна быть применима к любым изображениям, а не только художественным. Последние едва ли могут быть удачной отправной точкой для АФИ, поскольку это может привести к нормативной ориентации на эстетические режимы восприятия и оценивания. Поэтому можно говорить о значительной теоретической независимости АФИ от эстетики, которая лишь подчеркивается тем, что проблемы изобразительной репрезентации вызывают интерес не только у философов искусства, но и, к примеру, у представителей философии науки, когнитивных исследований и философии сознания. Время от времени взаимодействие с данными дисциплинами позиционируется в качестве приоритетного направления развития АФИ, потенциально способствующего легитимации ее достижений путем эмпирической поверки $[8,17,18]$. Но этот тренд не является центральным, несмотря на ряд работ, посвященных вопросам связи функционирования ментальных и изобразительных репрезентаций [19-21], роли изображений в научных практиках [22] или, например, использования сенсомоторных, телесно-ориентированных и иных нерепрезентационных теорий восприятия и сознания для объяснения изображений [23. Р. 82-113; 24].

Если же говорить об основной линии в развитии АФИ, то в ней можно наблюдать четко сформированный набор подходов, дискуссии между которыми определяют ее теоретическую идентичность. Придерживаясь приблизительной хронологии возникновения этих теорий, вкратце их можно представить следующим образом:

- Иллюзионистская теория Эрнста Гомбриха [25] сочетает перцептуализм с конвенционализмом [26]: изображения вызывают у зрителя иллюзию того, что их восприятие феноменологически идентично восприятию объектов лицом к лицу (это, в частности, обусловливает невозможность одновременного восприятия поверхности изображения и его объекта), но условием этой иллюзии являются репрезентативные схемы, неотделимые от социальных факторов и истории той или иной разновидности репрезентации.

- Структурные теории [7, 27, 28] имеют сравнительно мало общего со структурной лингвистикой и ее производными: они отталкиваются от концепции Нельсона Гудмена, в которой в качестве «ядра» изобразительной репрезентации постулировалась денотация. Указывая на конвенциональный 
характер восприятия изображений, Гудмен подчеркивал, что их необходимо исследовать в контексте символических систем, обладающих определенными семантическими и синтаксическими характеристиками.

- В предложенной Ричардом Уоллхеймом [29] теории «видения-в» (seeing-in) структурное различие между восприятием изображений и восприятием объектов лицом к лицу объясняется тем, что мы одновременно видим и изображенный объект, и поверхность изображения. Указание на «двусложHbiü» (twofold) характер восприятия изображений вызвало множество дискуссий и попыток уточнения и дальнейшего развития этой теории [30, 31].

- Подход Кендалла Уолтона [32] основан на понятии make-believe, т.e. воображаемой или игровой ситуации: в этой перспективе изображения выполняют функцию «подпорок» (props) для воображения реципиента, воображающего себя в ситуации действительного восприятия объекта изображения. Эта теория охватывает не только изобразительную репрезентацию, но и любые виды репрезентации в искусстве - с точки зрения Уолтона, неизменно имеющей фикциональный характер.

- Теории изображения, основывающиеся на концепции распознавания (Флинт Шиер [33], Доминик М. Лопес [3]), исходят из следующего аргумента: если у нас есть способность узнавать сильно изменившиеся за годы и десятки лет лица, то было бы по меньшей мере странно, если бы она «отказывала» в процессах понимания изображений. Тем самым подчеркивается дорефлексивная и непонятийная специфика этого понимания.

- В теориях Роберта Хопкинса [34] и Джона Хаймана [35] предпринимается попытка реабилитации миметических подходов к изобразительной репрезентации: сходство между изображением и объектом не является объективным, а реализуется в опыте реципиента.

Несмотря на неизбежную неполноту данного изложения, нетрудно заметить, что все подходы существенно различаются по своим методологиям, теоретическим акцентам, а порой и целям: как отмечают Кэтрин Эйбелл и Катерина Бантинаки, «среди философов нет и намека на консенсус относительно ключевых проблем философии изображений» [1. Р. 23]. Тем не менее можно выделить несколько точек пересечения между ними, указывающих на основные методологические и теоретические импликации АФИ.

Во-первых, практически все упомянутые теории нацелены на поиск «сильных» и вместе с тем емких теоретических объяснений пикториальной репрезентации, что делает их «жизнеспособными, но малоинформативными» [36]. Это может затруднять как раскрытие ряда теоретических нюансов, так и исследование отдельных изображений, а не только общих принципов их функционирования.

Во-вторых, несмотря на отсутствие общей методологической базы и разнообразие способов аргументации (от апелляций к обыденному опыту или мыслительных экспериментов до привлечения эмпирических исследований), в АФИ наблюдаются достаточно четкие стандарты доказательности и убедительности той или иной теории: в идеале она должна отличаться внутренней согласованностью и охватывать все разнообразие изображений, не противореча обыденным интуициям и не прибегая к редукционистским объяснениям.

В-третьих, для этих авторов характерен крайне ограниченный круг референций: к примеру, они практически не ссылаются на феноменологов или 
французских теоретиков образа. Некоторые из них, впрочем, сближаются с аналитической эстетикой, что зачастую приводит либо к неоправданному сужению предметного поля теории до фигуративных художественных изображений, либо к тому, что теория изобразительной репрезентации оказывается частным применением более широкой эстетической теории (как в случае с Н. Гудменом или К. Уолтоном).

В-четвертых, практически все упомянутые выше теории строятся вокруг новаторских концептов (таких как make-believe или двусложность). Не опираясь, по существу, на историю философии и подходя к феноменологическим или онтологическим проблемам «с нуля», представители АФИ стараются решать их посредством расширения, уточнения и нюансировки понятийного аппарата. За счет этого теоретические наработки данной школы могут представлять интерес и для исследователей, не разделяющих ее базовые философские допущения.

Наиболее оригинальную особенность АФИ можно продемонстрировать по контрасту с визуальными исследованиями (visual studies). В них зачастую ставится акцент на историчности и вариативности восприятия изображений, на их идеологических импликациях или, например, на зависящих от многочисленных контекстов практиках их производства, обращения и рецепции $[37,38]$. В АФИ все эти проблемы и факторы выносятся за скобки, что и обусловливает как ограничения, так и некоторые сильные стороны этой школы: игнорирование исторических, социальных и политических вопросов дает ее представителям возможность искать теоретический доступ к проблематике когнитивных, перцептивных, структурных и онтологических особенностей изобразительной репрезентации. Пожалуй, именно данное обстоятельство демонстрирует, что использование достижений этого направления исследований вне его контекста остро нуждается в их сопоставлении с альтернативными подходами.

Проведенный анализ позволяет заключить, что сформировавшийся собственный теоретический «канон» и ограниченность взаимодействий с другими дисциплинами и позволяют позиционировать АФИ как сообщество авторов, ищущих решения нескольких непростых теоретических вопросов в отсутствие собственных кафедр, журналов и других надежных маркеров дисциплинарной специализации. Как правило, эти вопросы четко ограничиваются областью изобразительной репрезентации (депикции) и затрагивают проблемы определения, онтологической двойственности, базового понимания и восприятия изображений. Установка на точность и изобретательность языка описания, методологическое разнообразие, строгость аргументации и неприятие нормативных эстетических подходов помогают многим представителям АФИ не только обеспечивать продуктивность такой «прицельной» теоретической работы, но и предлагать концепции, которые могут представлять эвристическую ценность и для других направлений исследования визуального и образного.

\section{Лuтература}

1. Abell C. \& Bantinaki K. (eds.) Philosophical Perspectives on Depiction. Oxford: Oxford University Press, 2010. $256 \mathrm{p}$.

2. Hecht H., Atherton M., Schwartz R. (eds.) Looking into Pictures: An Interdisciplinary Approach to Pictorial Space. Massachusetts: MIT Press, 2003. 417 p. 
3. Lopes D.M. Understanding Pictures. Oxford: Clarendon Press, 1996. 252 p.

4. Kulvicki J. Images. L., N. Y.: Routledge, 2014. 240 p.

5. Mitchell W. J. T. Iconology: Image, Text, Ideology. Chicago and London: University of Chicago Press, 1986. 225 p.

6. Peacocke C. Depiction // The Philosophical Review. 1987. Vol. 96, № 3. P. 383-410.

7. Goodman N. Languages of Art: An Approach to the Theory of Symbols. Indianapolis: BobbsMerill Company, 1968. $277 \mathrm{p}$.

8. Hopkins R. Pictures, Phenomenology and Cognitive Science // The Monist. 2003. Vol. 86, № 4. P. 653-675.

9. Polanyi M. What is a Painting? // The American Scholar. 1970. Vol. 39, № 4. P. 655-669.

10. Podro M. Depiction and the Golden Calf // Ibid. Philosophy and the visual arts: seeing and abstracting / Ed. by A. Harrison. Dordrecht: D. Reidel Publishing Company, 1997. P. 3-22.

11. Beardsley M.C. Aesthetics: Problems in the Philosophy of Criticism. 2nd edition. Indianapolis, Cambridge: Hackett, 1981. 614 p.

12. Gaut B., Lopes D.M. (eds.) The Routledge Companion to Aesthetics. L., N.Y.: Rotledge, 2001. $705 \mathrm{p}$.

13. Levinson J. (ed.) The Oxford Handbook of Aesthetics. Oxford: Oxford University Press, 2003. $825 \mathrm{p}$.

14. Carroll N. Philosophy of Art: A Contemporary Introduction. L., N.Y.: Rotledge, 2001. 286 p.

15. Eldridge R. An Introduction to the Philosophy of Art. Cambridge University Press, 2003. 285 p.

16. Atencia-Linares P. Pictures, Bytes and Values: An Interview with Dominic McIver Lopes // Postgraduate Journal Of Aesthetics. 2011, № 8 (2). [Online] Available from: http://www.pjaesthetics.org/index.php/pjaesthetics/article/view/10/8. (Accessed: 4h May 2017).

17. Lopes D.M. Pictures and the Representational Mind // The Monist. 2003. Vol. 86, № 4. P. 632-652.

18. Rollins M. Pictorial representation: When cognitive science meets aesthetics // Philosophical Psychology. 1999. Vol. 12. Issue 4. P. 387-413.

19. Currie G. Image and Mind: Film, philosophy and cognitive science. Cambridge: Cambridge University Press, $1995.332 \mathrm{p}$.

20. Currie G., Kieran M., Meskin A., Robson J. (Eds.) Aesthetics and the Sciences of Mind. Oxford: Oxford University Press, 2014. 272 p.

21. Schellekens E., Goldie P. (Eds.) The Aesthetic Mind: Philosophy and Psychology. Oxford: Oxford University Press, 2011. $420 \mathrm{p}$.

22. Kulvicki J. Knowing with Images: Medium and Message // Philosophy of Science. 2010. Vol. 77, № 2. P. 295-313.

23. Noe A. Varieties of Presence. London: Harvard University Press, 2012. 174 p.

24. Fingerhut J. Extended Imagery, Extended Access, Or Something Else? Pictures and the Extended Mind Hypothesis // Bildakt at the Warburg Institute. Berlin, 2014. P. 33-50.

25. Gombrich E.H. Art and Illusion: A Study in the Psychology of Pictorial Representation. L.: Phaidon Press, 1984. 402 p.

26. Bantinaki K. Pictorial Perception as Illusion // British Journal of Aesthetics. 2007. Vol. 47, № 3. P. 268-279. $274 \mathrm{p}$.

27. Kulvicki J. On Images: Their Structure and Content. Oxford: Oxford University Press, 2006.

28. Scholz O. A Solyd Sense of Syntax // Erkenntnis 2000. Vol. 52. P. 199-212.

29. Wollheim R. Seeing-as, Seeing-in and Pictorial Representation // Wollheim R. Art and its objects. Cambridge: Cambridge University Press, 1980. P. 205-226.

30. Bradley H. Reducing the Space of Seeing-In // British Journal of Aesthetics. 2015. Vol. 54. P. 409-424.

31. Newall M. Is Seeing-In a Transparency Effect? // British Journal of Aesthetics. 2015. Vol. 55, № 2. P. $131-156$.

32. Walton K.L. Mimesis as Make-Believe: On the Foundations of the Representational Arts. London: Harvard University Press, 1990. 480 p.

33. Schier F. Deeper into Pictures: An essay on pictorial representation. Cambridge: Cambridge University Press, 1986. 240 p.

34. Hopkins R. Picture, Image and Experience: A Philosophical Inquiry. Cambridge: Cambridge University Press, 1998.216 p.

35. Hyman J. The Objective Eye. Chicago: The University of Chicago Press, 2006. 300 p.

36. Lopes D.M. Beyond Art. Oxford: Clarendon Press, 2014. 240 p. 
37. Rose G. Visual Methodologies: An Introduction to Researching with Visual Materials. 4th edition. SAGE Publications, 2016. 456 p.

38. Sturken M., Cartwright L. Practices of Looking: An Introduction to Visual Culture. 2nd edition. Oxford: Oxford University Press, 2009. 496 p.

Nebolsin Daniil I. National Research University Higher School of Economics (Moscow, Russian Federation)

E-mail: daniil.nebolsin@gmail.com

DOI: $10.17223 / 1998863 X / 41 / 2$

ANALYTIC PHILOSOPHY OF DEPICTION: DISCIPLINARY AND THEORETICAL SPECIFICS

Key words: depiction, representation, pictures, analytical philosophy, image theory.

The aim of this article is to display the constitutive features of analytic philosophy of depiction (APD), a research field barely represented in Russian scientific literature. While sharing some core methodological implications and stylistic patterns with other subdivisions of analytic philosophy, APD tends to form rather enclosed philosophical community with its distinctive theoretical "canon" and highly specialized set of research questions. Philosophers of depiction explore the concept of picture rather than more conventional and broadly understood 'image'. From this perspective pictures are defined as two-dimensional objects simultaneously possessing some material vehicle (plane, surface) and some visual representational content. Philosophical understanding of pictures usually presupposes reflecting on problems of how pictorial representation works and in which respect it differs from other representational systems, of how ontological duality of depiction may be explained and of how to discern the conditions and singularities of pictorial experience and basic pictorial understanding. Some of these questions were initially posed by philosophers of art, but APD has already obtained theoretical independence from aesthetics at the current stage of its development. The core APD accounts include illusion theory (E.H. Gombrich), structural theories (N. Goodman, J. Kulvicki), seeing-in and twofoldness theory (R. Wollheim), make-believe theory (K. Walton), recognitional theories (F. Schier, D.M. Lopes) and experienced resemblance theories (J. Hyman, R. Hopkins). It is shown that despite their radical discrepancies, they share core standards of argumentation along with tendency to provide "strong" but concise explanations of depiction-related problems as well as to introduce novel, unorthodox notions in order to make their conceptual apparatuses more specified, fine-grained and nuanced. The most original and somewhat idiosyncratic feature of APD is that it generally avoids preoccupations with historical, political or ideological implications of pictures and social conditions of their production, circulation and reception. Hence any further applications of APD's theoretical accomplishments have a strong need to be supported by comparisons with other directions of research concerning the pictorial and the visual (such as interdisciplinary image studies, visual studies, iconology etc.).

\section{References}

1. Abell, C. \& Bantinaki, K. (eds) Philosophical Perspectives on Depiction. Oxford: Oxford University Press.

2. Hecht, H., Atherton, M. \& Schwartz, R. (eds.) Looking into Pictures: An Interdisciplinary Approach to Pictorial Space. Massachusetts: MIT Press.

3. Lopes, D.M. (1996) Understanding Pictures. Oxford: Clarendon Press.

4. Kulvicki, J. (2014) Images. London, New York: Routledge.

5. Mitchell, W.J.T. (1986) Iconology: Image, Text, Ideology. Chicago; London: University of Chicago Press.

6. Peacocke, C. (1987) Depiction. The Philosophical Review. 96(3). pp. 383-410.

7. Goodman, N. (1968) Languages of Art: An Approach to the Theory of Symbols. Indianapolis: Bobbs-Merill Company.

8. Hopkins, R. (2003) Pictures, Phenomenology and Cognitive Science. The Monist. 86(4). pp. 653-675. DOI: 10.5840 /monist2003

9. Polanyi, M. (1970) What is a Painting? The American Scholar. 39(4). pp. 655-669.

10. Podro, M. (1997) Depiction and the Golden Calf. In: Harrison, A. (ed.) Philosophy and the visual arts: seeing and abstracting. Dordrecht: D. Reidel Publishing Company. pp. 3-22.

11. Beardsley, M.C. (1981) Aesthetics: Problems in the Philosophy of Criticism. 2nd ed. Indianapolis, Cambridge: Hackett. 
12. Gaut, B. \& Lopes, D.M. (eds.) The Routledge Companion to Aesthetics. London, New York: Rotledge.

13. Levinson, J. (ed.) The Oxford Handbook of Aesthetics. Oxford: Oxford University Press.

14. Carroll, N. (2001) Philosophy of Art: A Contemporary Introduction. London, New York: Rotledge.

15. Eldridge, R. (2003) An Introduction to the Philosophy of Art. Cambridge University Press.

16. Atencia-Linares, P. (2011) Pictures, Bytes and Values: An Interview with Dominic McIver Lopes. Postgraduate Journal of Aesthetics. 8(2). [Online] Available from: http://www.pjaesthetics.org/index.php/pjaesthetics/article/view/10/8. (Accessed: 4th May 2017).

17. Lopes, D.M. (2003) Pictures and the Representational Mind. The Monist. 86(4). pp. 632-652. DOI: $10.2307 / 27903847$

18. Rollins, M. (1999) Pictorial representation: When cognitive science meets aesthetics. Philosophical Psychology. 12(4). pp. 387-413. DOI: 10.1080/095150899105657

19. Currie, G. (1995) Image and Mind: Film, philosophy and cognitive science. Cambridge: Cambridge University Press.

20. Currie, G., Kieran, M., Meskin, A. \& Robson, J. (eds) Aesthetics and the Sciences of Mind. Oxford: Oxford University Press.

21. Schellekens, E. \& Goldie, P. (eds) The Aesthetic Mind: Philosophy and Psychology. Oxford: Oxford University Press.

22. Kulvicki, J. (2010) Knowing with Images: Medium and Message. Philosophy of Science. 77(2). pp. 295-313. DOI: $10.1086 / 651321$

23. Noe, A. (2012) Varieties of Presence. London: Harvard University Press.

24. Fingerhut, J. (2014) Extended Imagery, Extended Access, Or Something Else? Pictures and the Extended Mind Hypothesis. Berlin: Bildakt at the Warburg Institute. pp. 33-50.

25. Gombrich, E.H. (1984) Art and Illusion: A Study in the Psychology of Pictorial Representation. London: Phaidon Press. $268-279$.

26. Bantinaki, K. (2007) Pictorial Perception as Illusion. British Journal of Aesthetics. 47(3). pp.

27. Kulvicki, J. (2006) On Images: Their Structure and Content. Oxford: Oxford University Press.

28. Scholz, O. (2000) A Solyd Sense of Syntax. Erkenntnis. 52. pp. 199-212.

29. Wollheim, R. (1980) Art and its Objects. Cambridge: Cambridge University Press. pp. 205226.

30. Bradley, H. (2015) Reducing the Space of Seeing-In. British Journal of Aesthetics. 54(4). pp. 409-424.

31. Newall, M. (2015) Is Seeing-In a Transparency Effect? British Journal of Aesthetics. 55(2). pp. 131-156.

32. Walton, K.L. (1990) Mimesis as Make-Believe: On the Foundations of the Representational Arts. London: Harvard University Press.

33. Schier, F. (1986) Deeper into Pictures: An essay on pictorial representation. Cambridge: Cambridge University Press.

34. Hopkins, R. (1998) Picture, Image and Experience: A Philosophical Inquiry. Cambridge: Cambridge University Press.

35. Hyman, J. (2006) The Objective Eye. Chicago: The University of Chicago Press.

36. Lopes, D.M. (2014) Beyond Art. Oxford: Clarendon Press.

37. Rose, G. (2016) Visual Methodologies: An Introduction to Researching with Visual Materials. 4th ed. SAGE Publications.

38. Sturken, M. \& Cartwright, L. (2009) Practices of Looking: An Introduction to Visual Culture. 2nd ed. Oxford: Oxford University Press. 